\title{
Appraisal Theories for Emotion Classification in Text
}

\author{
Jan Hofmann ${ }^{1}$, Enrica Troiano ${ }^{1}$, Kai Sassenberg ${ }^{2,3}$, and Roman Klinger ${ }^{1}$ \\ ${ }^{1}$ Institut für Maschinelle Sprachverarbeitung, University of Stuttgart, Germany \\ ${ }^{2}$ Leibniz-Institut für Wissensmedien, Tübingen, Germany \\ ${ }^{3}$ University of Tübingen, Germany \\ \{jan.hofmann, enrica.troiano, roman.klinger\}eims.uni-stuttgart.de \\ k. sassenbergeiwm-tuebingen. de
}

\begin{abstract}
Automatic emotion categorization has been predominantly formulated as text classification in which textual units are assigned to an emotion from a predefined inventory, for instance following the fundamental emotion classes proposed by Paul Ekman (fear, joy, anger, disgust, sadness, surprise) or Robert Plutchik (adding trust, anticipation). This approach ignores existing psychological theories to some degree, which provide explanations regarding the perception of events. For instance, the description that somebody discovers a snake is associated with fear, based on the appraisal as being an unpleasant and non-controllable situation. This emotion reconstruction is even possible without having access to explicit reports of a subjective feeling (for instance expressing this with the words "I am afraid."). Automatic classification approaches therefore need to learn properties of events as latent variables (for instance that the uncertainty and the mental or physical effort associated with the encounter of a snake leads to fear). With this paper, we propose to make such interpretations of events explicit, following theories of cognitive appraisal of events, and show their potential for emotion classification when being encoded in classification models. Our results show that high quality appraisal dimension assignments in event descriptions lead to an improvement in the classification of discrete emotion categories. We make our corpus of appraisal-annotated emotion-associated event descriptions publicly available.
\end{abstract}

\section{Introduction}

The task of emotion analysis is commonly formulated as classification or regression in which textual units (documents, paragraphs, sentences, words) are mapped to a predefined reference system, for instance the sets of fundamental emotions fear, anger, joy, surprise, disgust, and sadness proposed by Ekman (1999), or by Plutchik (2001), which includes also trust and anticipation. Machine learning-based models need to figure out which words point to a particular emotion experienced by a reader, by the author of a text, or a character in it. Depending on the resource which has been annotated, the description of an emotion experience can vary. On Twitter, for instance, other than direct reports of an emotion state ("I feel depressed"), hashtags are used as emotion labels to enrich the description of events and stances ("I just got my exam result \#sad"). In news articles, emotional events are sometimes explicitly mentioned ("couple infuriate officials" (Bostan et al., 2020)) and other times require world knowledge ("Tom Cruise and Katie Holmes set wedding date", labeled as surprise (Strapparava and Mihalcea, 2007)). In literature, a sequence of events which forms the narrative leads to an emotion in the reader. In this paper, we focus on those texts which communicate emotions without an explicit emotion word, but rather describe events for which an emotion association is evident.

Such textual examples became popular in natural language processing research with the use of the data generated in the ISEAR project (Scherer and Wallbott, 1997). The project led to a dataset of descriptions of events triggering specific affective states, which was originally collected to study event interpretations with a psychological focus. In text analysis, to infer the emotion felt by the writers of those reports, an

\footnotetext{
This work is licensed under a Creative Commons Attribution 4.0 International License. License details: http:// creativecommons.org/licenses/by/4.0/.
} 
event interpretation needs to be accomplished. For instance, in the text "When a car is overtaking another and I am forced to drive off the road", the model needs to associate the event with fear.

To date, nearly all computational approaches that associate text with emotions are agnostic to the way in which emotions are communicated, they do "not know" how to interpret events, but, presumably, they purely learn word associations instead of actual event interpretations. One might argue that approaches predicting fine-grained dimensions of affect, namely arousal and valence, actually tackle this problem (Buechel and Hahn, 2017; Preoţiuc-Pietro et al., 2016). However, these typically do not infer downstream emotion categories. Further, particularly regarding events, psychological theories offer more detailed information. As an example, the emotion component model (Scherer, 2005) advocates that cognitive appraisal dimensions underly discrete emotion classes (Smith and Ellsworth, 1985). These appraisal dimensions ${ }^{1}$ evaluate (1) how pleasant an event is (pleasantness, likely to be associated with joy, but unlikely to appear with disgust), (2) how much effort an event can be expected to cause (anticipated effort, likely to be high when anger or fear is experienced), (3) how certain the experiencer is in a specific situation (certainty, low, e.g., in the context of hope or surprise), (4) how much attention is devoted to the event (attention, likely to be low, e.g., in the case of boredom or disgust), (5) how much responsibility the experiencer of the emotion holds for what has happened (self-other responsibility/control, high for feeling challenged or pride), and (6) how much the experiencer has control over the situation (situational control, low in the case of anger).

Despite their richness, cognitive theories of appraisal and their empirical results have not been exploited for emotion prediction in text yet. We fill this gap with this paper and analyze the relation between appraisal dimensions and emotion categories in a text classification setting. We post-annotate an English emotion corpus of self-reports of emotion events (Troiano et al., 2019), which already contains annotations related to the emotions of anger, disgust, fear, guilt, joy, sadness, and shame, and add the appraisal dimensions by Smith and Ellsworth (1985) mentioned above. Further, we analyze if an automatic prediction of these dimensions from text is possible with standard neural methods, and if these predictions contribute to emotion classifications. Our main contributions are: (1) the first event-centered text corpus annotated with appraisal dimensions; (2) the evaluation of how well text classification models can recognize these appraisal dimensions; (3) we show emotion classification benefits from the information of appraisal dimensions, when high quality predictions of these are available. Further, (4), we replicate the study by Smith and Ellsworth (1985) from a CL/NLP perspective, based on textual event descriptions.

\section{Background on Emotion Psychology and Analysis}

\subsection{Emotion and Affect Theories}

As a component of humans' life, emotions have been thoroughly studied in the field of psychology, where they are generally deemed responses to salient events. The debates surrounding their definition, however, has never come to a consensus, producing a varied literature on the topic. This has a clear implication for computational emotion analyses, for they must choose and follow one of the available psychological theories in order to motivate the emotion phenomenon that they research in language.

Some of such theories focus on the evolutionary function of emotions, and accordingly, on their link to actions (Izard, 1971; Tooby and Cosmides, 2008). The core idea is that emotions help humans accomplish every-day life tasks and communicate socially relevant information by triggering specific physiological symptoms. In particular, there are patterns of behaviour (e.g., smiling) that reflect discrete emotion terms (e.g., joy), which suggests that emotional states can be grouped based on a few natural language categories. One of the most popular sources for a set of fundamental emotions is the theory by Ekman (1992). Ekman studied the relation between emotions and both culture and facial expressions: he claimed that the set of fundamental emotions, namely, anger, disgust, fear, joy, sadness, and surprise can be distinguished by facial muscular movements across cultures (which is partially doubted these days, Gendron et al. (2014)). As an addition to this model, Plutchik (2001) makes explicit the assumption that different fundamental emotions can occur together, for instance trust and joy, which is the case when love is experienced. Such emotion mixtures, as well as an opposition between anger and fear, joy and sadness, surprise and

\footnotetext{
${ }^{1}$ The examples follow the results by Smith and Ellsworth (1985), an excerpt is shown in Table 1.
} 
anticipation, trust and disgust, has been included in this model. In natural language processing, mostly a set of four to eight fundamental emotions is used, where anger, fear, joy, and sadness are shared by most approaches (an exception with 24 emotion classes is Abdul-Mageed and Ungar (2017)).

A diametrically opposite view is held by the constructivist tradition (Averill, 1980; Oatley, 1993; Barrett and Russell, 2015), in which actions and physiological changes are the building blocks that construct emotions, rather than their direct effect (Feldman Barrett, 2006). Feeling an emotion means categorizing the fluctuations of an affect system along some components. For instance, the affect components valence (degree of polarity), arousal (degree of excitement), and dominance (degree of control over a situation) (Posner et al., 2005) are used as dimensions to describe affect experiences in a 3-dimensional space, which can then be mapped to discrete emotion categories.

\subsection{Theories of Cognitive Appraisal}

Extensions to this model in terms of underlying components are the works of Scherer (1982), Smith and Ellsworth (1985) and Oatley and Johnson-Laird (1987), who qualified emotions as component processes that arise to face salient circumstances: an emotion is an "episode of interrelated, synchronized changes in the states of all or most of the five organismic subsystems in response to the evaluation of an external or internal stimulus-event as relevant to major concerns of the organism" (Scherer et al., 2001). According to this view, there is an appraisal, that is, an information processing component, which enables people to determine the significance of a situation with respect to their needs and values. In the context of this appraisal (e.g., judging a snake as dangerous), the resources of four other components are mobilitated to deal with the situation. These are, next to the cognitive component (appraisal), a neurophysiological component (bodily symptoms), a motivational component (action tendencies), a motor expression (facial and vocal expression), and a subjective feeling component (emotional experience) (Scherer, 2005, Table 1).

While the notions of subjective experience and bodily symptoms were common to other emotion theories, appraisal represents a novelty that fills in some shortcomings of basic models. First, it explains how emotions are elicited. The origin of emotions is to be seen in the stimulus as appraised rather than in the stimulus as such. Second, appraisals provide a structured account for the differences among emotions. For instance, anger and fear are experienced when the evaluation of a negative event attributes it to external factors, whereas guilt and shame are felt if the causes of such event are identified in the self, as stable and uncontrollable personality traits, like in the case of shame (e.g., "I'm dumb"), or unstable and controllable behaviours for guilt (e.g., "I did not observe the speed limit") (Tracy and Robins, 2006).

We argue in this paper that this makes appraisals particularly useful for natural language processing, because they both provide a framework for research and represent a way of enriching existing data. As a matter of fact, few dimensions are sufficient to explain emotions based on cognitive appraisal. Smith and Ellsworth (1985) explain 15 emotions by leveraging pleasantness (polarity), self-other responsibility/control (for initiating the situation), certainty (about what is going on), attention (whether the emotion stimulus is worth attending), anticipated effort (the amount of physical or mental activation before the stimulus), and situational control (the ability to cope with the situation). Compared to the valence-arousal-dominance model, where it is left unclear if the polarity dimension refers to a quality of the emotion stimulus or a quality of the feeling (Scherer, 2005), all these dimensions are unambiguously event-directed. In this paper, we focus on modelling the cognitive components described by Smith and Ellsworth (1985). We show their main findings in Table 1, limited to the emotions that are available in the corpus we use.

\subsection{Automatic Emotion Classification}

Previous work on emotion analysis in natural language processing focuses either on resource creation or on emotion classification for a specific task and domain. On the side of resource creation, the early and influential work of Pennebaker et al. (2001) is a dictionary of words being associated with different psychologically relevant categories, including a subset of emotions. Later, Strapparava and Valitutti (2004) made WordNet Affect available to target word classes and differences regarding their emotional connotation, Mohammad and Turney (2012) released the NRC dictionary with more than 14,000 words for a set of discrete emotion classes, and a valence-arousal-dominance dictionary was provided by Mohammad 


\begin{tabular}{|c|c|c|c|c|c|c|}
\hline Emotion & Unpleasant & Responsibility & Uncertainty & Attention & Effort & Control \\
\hline Happiness & -1.46 & 0.09 & -0.46 & 0.15 & -0.33 & -0.21 \\
\hline Sadness & 0.87 & -0.36 & 0.00 & -0.21 & -0.14 & 1.15 \\
\hline Anger & 0.85 & -0.94 & -0.29 & 0.12 & 0.53 & -0.96 \\
\hline Fear & 0.44 & -0.17 & 0.73 & 0.03 & 0.63 & 0.59 \\
\hline Disgust & 0.38 & -0.50 & -0.39 & -0.96 & 0.06 & -0.19 \\
\hline Shame & 0.73 & 1.31 & 0.21 & -0.11 & 0.07 & -0.07 \\
\hline Guilt & 0.60 & 1.31 & -0.15 & -0.36 & 0.00 & -0.29 \\
\hline
\end{tabular}

Table 1: The locations of emotions along appraisal dimensions (according to a principle component analysis) as published by Smith and Ellsworth (1985), Table 6, filtered to those emotions which are available in the text corpus we use.

(2018). Buechel et al. (2016) have developed a methodological framework to adapt existing affect lexicons to specific use cases. Other than dictionaries, emotion analysis relies on labeled corpora. Some of them include information relative to valence and arousal (Buechel and Hahn, 2017; Preotiuc-Pietro et al., 2016), but the majority of resources use discrete emotion classes, for instance to label fairy tales (Alm et al., 2005), blogs (Aman and Szpakowicz, 2007), tweets (Mohammad et al., 2017; Schuff et al., 2017; Mohammad, 2012; Mohammad and Bravo-Marquez, 2017; Klinger et al., 2018), Facebook posts (Preoţiuc-Pietro et al., 2016), news headlines (Strapparava and Mihalcea, 2007), dialogues (Li et al., 2017), literary texts (Kim et al., 2017), or self reports on emotion events (Scherer and Wallbott, 1997; Troiano et al., 2019). We point the reader to the survey by Bostan and Klinger (2018) for a more an overview.

Most automatic methods that assign labels to text rely on machine learning (Alm et al., 2005; Aman and Szpakowicz, 2007; Schuff et al., 2017, i.a.). Recent shared tasks showed an increase in transfer learning from generic representations (Klinger et al., 2018; Mohammad et al., 2018; Mohammad and Bravo-Marquez, 2017). Felbo et al. (2017) proposed to use emoji representations for pretraining, and Cevher et al. (2019) performed pretraining on existing emotion corpora followed by fine-tuning for a specific domain for which only little training data was available.

We are only aware of one preliminary study which considered appraisal dimensions to improve textbased emotion prediction, namely Campero et al. (2017). In their study, subjects labeled 200 stories with 38 appraisal features (which remain unmentioned), to evaluate if a text-based representation adds on top of an fMRI-based classification. Apart from this study, all previous machine learning-based approaches used models to predict emotions or affect values directly from text, without any access to appraisal dimensions. Only a couple of works incorporated cognitive components, for instance those coming from the OCC model (named after the authors Ortony, Clore and Collins' initials), which sees every appraisal as an evaluation of the pleasantness of events, objects, or actions with respect to one's goals, tastes or behavioural and moral standards (Clore and Ortony, 2013). Based on the OCC model, Shaikh et al. (2009) devised a rule-based approach to interpret text. They did not explicitly formulate their model following appraisal theories, but they moved towards a cognitively-motivated interpretation of events and interpersonal descriptions. Others have adopted patterns of appraisal to predict the emotions triggered by actions, as described in a text. Specifically, Balahur et al. (2011) and Balahur et al. (2012) have created EmotiNet, a knowledge base of action chains that includes information about the elements on which the appraisal is performed within an affective situation, namely, the agent, the action and the object involved in a chain. We share their motivation to delve into event representations based on the descriptions of their experiencers. Unlike their work, ours explicitly encodes appraisal dimensions and uses the classification into these categories for emotion prediction.

We are not aware of any textual corpus annotated with appraisals according to emotion theories, or associated computational models. However, some research exists which studies these theories with added value for computational models. Scherer et al. (2019), e.g., explicitly encode realizations of appraisals of events and compare those between production and perception of facial expressions. Marsella and Gratch (2009) model emotions in video sequences computationally, based on appraisals of events. Broekens et al. (2008) inform intelligent agents about emotions based on formalizations of cognitive appraisal. Further, in 
Scherer et al. (2010), the chapter authored by Marsella et al. (2010) argues for an integrated computational approach of emotions which also embeds appraisal theories.

\section{Corpus}

The main objective of this study is to understand the relation between appraisal dimensions and emotion categories. Therefore, we build appraisal annotations on top of enISEAR, an existing corpus of 1001 English event descriptions which are already labeled with the discrete categories of anger, disgust, fear, guilt, joy, sadness, and shame (Troiano et al., 2019). Each instance has been generated by a crowdworker on the platform FigureEight by completing the sentence "I feel [emotion name], when ...". This corpus has an advantage over the original ISEAR resource because it has a German counterpart which can be used in further studies; moreover, its emotion labels have been intersubjectively validated. The corpus, annotations, and our implementations are available at http://www.ims.uni-stuttgart.de/ data/appraisalemotion.

\subsection{Annotation}

One presumable challenge in the post-annotation of events regarding the appraisal dimensions is that our annotators do not have access to the private state of the event experiencers. However, under the assumption that events are perceived similarly in subjective feeling and evaluated comparably based on cognitive appraisal, we assume that this is not a major flaw in the design of the study. An alternative would have been to perform the text generation task as Troiano et al. (2019) did, but asking the authors of event descriptions for their appraisal in addition. We opted against such procedure as it would have meant to reproduce an existing study in addition to our research goal.

For the post-labeling of enISEAR, we aimed at formulating unambiguous and intuitive descriptions of appraisal dimensions, which would be faithful to those in Smith and Ellsworth (1985). As opposed to the subjects of their study, however, our annotators had to judge events that they did not personally experience. For this reason, we simplified our annotation guidelines in two respects. First, we opted for a binary setting, while Smith and Ellsworth (1985) used continuous scales to rate discrete emotion categories on the appraisal dimensions. Second, we split control into Control and Circumstance (i.e., self and situational control), in line with the discussion of this variable by Smith and Ellsworth (1985, p. 824f.), while retaining the category of responsibility. This was motivated by the observation of a low inter-annotator agreement in preliminary annotation rounds and a series of discussions that revealed the difficulty for annotators to separate the concepts of responsibility and self control. Then, the annotators were instructed to read an event description, without having access to the emotion label, and to answer the following questions:

Most probably, at the time when the event happened, the writer...

- ... wanted to devote further attention to the event. (Attention)

- ... was certain about what was happening. (Certainty)

- ... had to expend mental or physical effort to deal with the situation. (Effort)

- ... found that the event was pleasant. (Pleasantness)

- ... was responsible for the situation. (Responsibility)

- ... found that he/she was in control of the situation. (Control)

- ... found that the event could not have been changed or influenced by anyone. (Circumstance)

Each event description from enISEAR was judged by three annotators between the age of 26 and 29 . One of them is a female Ph.D. student of computational linguistics, the others are male graduate students of software engineering. Two of the annotators are co-authors of this paper. The judges familiarised themselves with their task through four training iterations. At every iteration, we hand-picked 15-20 samples from the ISEAR dataset (Scherer and Wallbott, 1997), such that instances used for training would not be seen during the actual annotation, but had a comparable structure. Dissimilarities in the annotation were discussed in face-to-face meetings and the annotation guideline was refined.

The agreement improved from $\kappa=0.62$ to 0.67 in the four iterations. In one of them, we experimented with giving access to the emotion label, which lead to a large improvement in agreement $(\kappa=0.83)$. 


\begin{tabular}{|c|c|c|c|c|c|c|c|c|}
\hline \multirow[b]{3}{*}{ Appraisal Dimension } & \multicolumn{8}{|c|}{ Cohen's $\kappa$} \\
\hline & \multicolumn{4}{|c|}{ between annotators } & \multicolumn{4}{|c|}{ annotator-majority } \\
\hline & A1/A2 & A1/A3 & A2/A3 & avg. & A1 & A2 & A3 & avg. \\
\hline Attentional Activity & .28 & .24 & .41 & .31 & .50 & .76 & .66 & .64 \\
\hline Certainty & .41 & .23 & .29 & .31 & .62 & .77 & .46 & .62 \\
\hline Anticipated Effort & .38 & .33 & .26 & .32 & .69 & .67 & .62 & .66 \\
\hline Pleasantness & .89 & .88 & .90 & .89 & .93 & .96 & .94 & .94 \\
\hline Responsibility & .68 & .57 & .63 & .63 & .80 & .88 & .76 & .81 \\
\hline Control & .65 & .56 & .52 & .58 & .84 & .81 & .70 & .78 \\
\hline Circumstance & .52 & .32 & .28 & .37 & .80 & .69 & .49 & .66 \\
\hline Average & .59 & .48 & .52 & .53 & .77 & .82 & .70 & .76 \\
\hline
\end{tabular}

Table 2: Cohen's $\kappa$ between all annotator pairs and between each annotator and the majority vote.

\begin{tabular}{|c|c|c|c|c|c|c|c|c|c|c|c|c|c|c|}
\hline \multirow{3}{*}{$\frac{\text { Emotion }}{\text { Anger }}$} & \multicolumn{14}{|c|}{ Appraisal Dimension } \\
\hline & \multicolumn{2}{|c|}{ Attention } & \multicolumn{2}{|c|}{ Certainty } & \multicolumn{2}{|c|}{ Effort } & \multicolumn{2}{|c|}{ Pleasant } & \multicolumn{2}{|c|}{ Respons. } & \multicolumn{2}{|c|}{ Control } & \multicolumn{2}{|c|}{ Circum. } \\
\hline & 129 & .90 & 119 & .83 & 60 & .42 & 0 & .00 & 9 & .06 & 1 & .01 & 5 & .03 \\
\hline Disgust & 67 & .47 & 134 & .94 & 40 & .28 & 2 & .01 & 14 & .10 & 11 & .08 & 24 & .17 \\
\hline Fear & 129 & .90 & 13 & .09 & 121 & .85 & 4 & .03 & 43 & .30 & 18 & .13 & 66 & .46 \\
\hline Guilt & 55 & .38 & 132 & .92 & 36 & .25 & 0 & .00 & 133 & .93 & 88 & .62 & 11 & .08 \\
\hline Joy & 139 & .97 & 140 & .98 & 4 & .03 & 141 & .99 & 65 & .45 & 41 & .29 & 25 & .17 \\
\hline Sadness & 122 & .85 & 112 & .78 & 88 & .62 & 1 & .01 & 7 & .05 & 2 & .01 & 97 & .68 \\
\hline Shame & 32 & .22 & 111 & .78 & 51 & .36 & 1 & .01 & 106 & .74 & 67 & .47 & 12 & .08 \\
\hline Total & 673 & & 761 & & 400 & & 149 & & 377 & & 228 & & 240 & \\
\hline
\end{tabular}

Table 3: Instance counts and ratios across emotions and appraisal annotations.

Nevertheless, we decided to continue without this information, in order to evaluate the annotator's performance in a similar setting as we evaluate the automatic model - to predict appraisal for emotion classification. We show the pairwise inter-annotator scores of the final set in Table 2. The agreement scores between the different annotator pairs are comparable.

These scores tell us that rating appraisal dimensions for given events is challenging, and its difficulty varies depending on the categories. Given the comparably low agreement obtained for a subset of dimensions, we opt for a "crowd-sourcing"-like aggregation by taking the majority vote to form the final annotation, included in Table 2, on the right side of the table. We observe that the agreement between majority vote and each annotator is constantly above $\kappa=.62$, which is an acceptable agreement (avg. $\kappa=.76$ ).

\subsection{Analysis}

In Table 3 are the cooccurrence counts across emotion and appraisal dimension pairs, as well as the relative counts normalized by emotion (enISEAR provides 143 descriptions per emotion). The most frequently annotated class is certainty, followed by attention. Appraisal dimensions are differently distributed across emotions: anger and fear require attention, guilt and shame do not; disgust and anger show the highest association with certainty, in opposition to fear. Responsibility and control play the biggest role in guilt and shame, while joy, non-surprisingly, strongly relates to pleasantness. Fear has a clear link with anticipated effort and, together with sadness, it is characterized by the inability to control the circumstance.

These numbers are particularly interesting in comparison with the findings of Smith and Ellsworth (1985), who report the average scores along the appraisal dimensions (based on a principle component analysis) for each emotion ${ }^{2}$. Results are consistent in most cases. For instance, joy (or happiness in Table 1) stands out as highly pleasant and barely related to anticipated effort. Self responsibility is lowest in anger, an emotion that arises when blame is externalized, and mostly present in shame and guilt, which derive from blaming the self (Tracy and Robins, 2006). These two are also the emotions that annotators

\footnotetext{
${ }^{2}$ We report the subset of emotions that overlap with ours. Also note that their "Control" corresponds to our "Circum.".
} 
associated with control more than others. Attention is prominent for events that elicited anger and which were under the control of others, as suggested by the low situational control. The highest situational control, on the contrary, appears with the data points labeled as fear, also characterized by a strong feeling of uncertainty and anticipated effort. There are also dissimilarities between the two tables, like the level of attention, reaching the lowest score for disgust in their study and not in ours. They also find that situational control is a stronger indicator for shame than for guilt, while anticipated effort is more marked in our sadness-related events than theirs.

These differences may partly be data-specific, partly due to the type of metrics shown in the tables. Most importantly, they can be traced to the annotation setup: while their subjects recalled and appraised personal events, our annotators evaluated the descriptions of events that are foreign to them.

It should be noted that for a reader/annotator it is challenging to impersonate in the writer: although some events have a shared understanding (e.g., "I passed the exam" is most likely appraised as pleasantness), others are tied to one's personal background, values and preferences. This may represent a source of disaccord both between the tables, among the annotators, and with the emotion labels themselves (e.g., "I felt ... when my mom offered me curry" has a pleasant gold label, while the original author meant it as a negative emotion, namely disgust).

\section{Experiments}

We now move to our evaluation if automatic methods to recognize emotions can benefit from being informed about appraisal dimensions explicitly. We first describe our models and how we address our research questions and then turn to the results.

\subsection{Model Configuration}

Figure 1 illustrates the four different tasks addressed by our models. Task $\mathbf{T} \rightarrow \mathbf{E}$ is the prediction of emotions from text, namely the standard setting in nearly all previous work of emotion analysis. We use a convolutional neural network (CNN) inspired by Kim (2014), with pretrained GloVe (Glove840B) as a 300-dimensional embedding layer (Pennington et al., 2014) ${ }^{3}$ with convolution filter sizes of 2, 3, and 4 with a ReLu activation function (Nair and Hinton, 2010), followed by a max pooling layer of length 2 and a dropout of 0.5 followed by another dense layer.

As another model to predict emotions, we use a pipeline based on two steps, one to detect the appraisal from text, and the second to assign the appropriate emotion to the appraisal. We refer to Task $\mathbf{T} \rightarrow \mathbf{A}$ as the step of identifying appraisal from text. We use the model configuration of Task $\mathrm{T} \rightarrow \mathrm{E}$, except for the sigmoid activation function and binary cross entropy loss instead of softmax and cross-entropy loss. As a second step, Task $\mathbf{A} \rightarrow \mathbf{E}$ predicts emotion from appraisal. The features are seven boolean variables corresponding to the appraisal dimensions. We use a neural network with two hidden layers with ReLU activation, followed by a dropout of $0.5 .^{4}$

A disadvantage of the pipeline setting could be that the emotion prediction needs to handle propagated errors from the first step, and that the first step cannot benefit from what the second model learns. Therefore, we compare the pipeline setting $(\mathrm{T} \rightarrow \mathrm{A}, \mathrm{A} \rightarrow \mathrm{E}$ ) with a multi-task learning setting $(\mathbf{T} \rightarrow \mathbf{A} / \mathbf{E})$. The model is similar to Task $\mathrm{T} \rightarrow \mathrm{E}$. The convolutional layer is shared by the tasks of predicting emotions from text and predicting appraisal from text and we use two output layers, one for emotion predictions with softmax activation and one for appraisal predictions with sigmoid activation.

\subsection{Results}

We perform each experiment in a repeated $10 \times 10$-fold cross-validation setting and report average results. All partitions of training and test sets are the same across all experiments.

Experiment 1 (Appraisal Prediction, $\mathbf{T} \rightarrow \mathbf{A}$ ) aims at understanding how well appraisal dimensions can be predicted from text. Emotion classification is an established task, and one might have some

\footnotetext{
${ }^{3} \mathrm{https}: / /$ nlp.stanford.edu/projects/glove/

${ }^{4}$ We do not perform any further hyperparameter search. We also compared all model configurations to MaxEnt models to ensure that we do not suffer from overfitting due to a large number of parameters, in comparison to the limited training data. In all settings, the neural models were superior. We therefore limit our explanations to those.
} 

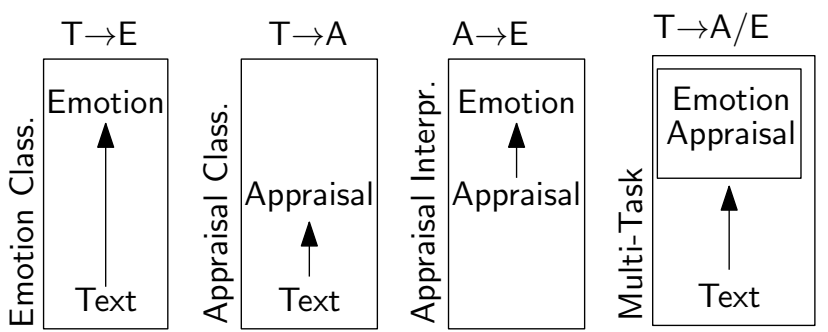

Figure 1: Tasks investigated in experiments on appraisaldriven emotion analysis.

\begin{tabular}{lcccc}
\hline & & \multicolumn{3}{c}{$\mathrm{T} \rightarrow \mathrm{A}$} \\
\cline { 3 - 5 } Appraisal & & $\mathrm{P}$ & $\mathrm{R}$ & $\mathrm{F}_{1}$ \\
\cline { 1 - 2 } \cline { 4 - 5 } Attention & & 81 & 84 & 82 \\
Certainty & & 84 & 86 & 85 \\
Effort & & 68 & 68 & 68 \\
Pleasantness & & 79 & 63 & 70 \\
Responsibility & & 74 & 68 & 71 \\
Control & & 63 & 49 & 55 \\
Circumstance & & 65 & 58 & 61 \\
\cline { 1 - 2 } Macro avg. & & 73 & 68 & 70 \\
Micro avg. & & 77 & 74 & 75 \\
\hline
\end{tabular}

Table 4: Classifier performance on predicting appraisal dimensions.

\begin{tabular}{|c|c|c|c|c|c|c|c|c|c|c|c|c|c|c|c|c|c|c|}
\hline \multirow[b]{3}{*}{ Emotion } & & & & & & & & & & & & & \multicolumn{6}{|c|}{ Oracle Ensembles } \\
\hline & \multicolumn{3}{|c|}{$\mathrm{T} \rightarrow \mathrm{E}$} & \multicolumn{3}{|c|}{$\mathrm{T} \rightarrow \mathrm{A}, \mathrm{A} \rightarrow \mathrm{E}$} & \multicolumn{3}{|c|}{$\mathrm{A} \rightarrow \mathrm{E}$ (Gold) } & \multicolumn{3}{|c|}{$\mathrm{T} \rightarrow \mathrm{A} / \mathrm{E}$} & \multicolumn{3}{|c|}{$\mathrm{T} \rightarrow \mathrm{A} \rightarrow \mathrm{E}+\mathrm{T} \rightarrow \mathrm{E}$} & \multicolumn{3}{|c|}{$\mathrm{T} \rightarrow \mathrm{A} / \mathrm{E}+\mathrm{T} \rightarrow \mathrm{E}$} \\
\hline & $\mathrm{P}$ & $\mathrm{R}$ & $\overline{F_{1}}$ & $\mathrm{P}$ & $\mathrm{R}$ & $\overline{F_{1}}$ & $\mathrm{P}$ & $\mathrm{R}$ & $\overline{F_{1}}$ & $\mathrm{P}$ & $\mathrm{R}$ & $\overline{F_{1}}$ & $\mathrm{P}$ & $\mathrm{R}$ & $\mathrm{F}_{1}$ & $\mathrm{P}$ & $\mathrm{R}$ & $\mathrm{F}_{1}$ \\
\hline Anger & 51 & 52 & 52 & 34 & 62 & 44 & 55 & 71 & 62 & 51 & 52 & 52 & 66 & 81 & 73 & 59 & 59 & 59 \\
\hline Disgust & 65 & 63 & 64 & 59 & 34 & 43 & 53 & 48 & 51 & 64 & 64 & 64 & 78 & 68 & 73 & 69 & 66 & 67 \\
\hline Fear & 69 & 71 & 70 & 55 & 55 & 55 & 79 & 78 & 78 & 70 & 68 & 69 & 76 & 77 & 77 & 73 & 75 & 74 \\
\hline Guilt & 47 & 42 & 44 & 38 & 50 & 43 & 57 & 70 & 63 & 45 & 42 & 44 & 60 & 63 & 62 & 58 & 54 & 56 \\
\hline Joy & 74 & 80 & 77 & 77 & 69 & 72 & 94 & 98 & 96 & 77 & 77 & 77 & 79 & 80 & 80 & 79 & 85 & 82 \\
\hline Sadness & 69 & 67 & 68 & 58 & 40 & 47 & 69 & 63 & 66 & 68 & 68 & 68 & 74 & 70 & 72 & 73 & 71 & 72 \\
\hline Shame & 44 & 45 & 45 & 36 & 24 & 29 & 56 & 35 & 43 & 43 & 43 & 43 & 58 & 51 & 54 & 51 & 52 & 52 \\
\hline Macro avg. & 60 & 60 & 60 & 51 & 48 & 48 & 66 & 66 & 65 & 60 & 59 & 59 & 70 & 70 & 70 & 66 & 66 & 66 \\
\hline Micro avg. & & & 60 & & & 48 & & & 66 & & & 59 & & & 70 & & & 66 \\
\hline
\end{tabular}

Table 5: Comparison of the Text-to-Emotion baseline $(\mathrm{T} \rightarrow \mathrm{E})$ with the performance of first prediction appraisal followed by emotion analysis $(\mathrm{T} \rightarrow \mathrm{A}, \mathrm{A} \rightarrow \mathrm{E})$ and the multi-task setting $(\mathrm{T} \rightarrow \mathrm{A} / \mathrm{E})$. The oracle consists of a combination of two models and is informed which model is more likely to make the correct prediction.

intuition on the expected performance with a given data set, but the prediction of appraisal dimensions has never been performed before. Hence, we report precision, recall, and $\mathrm{F}_{1}$ for each appraisal component considered in Table 4 . The prediction of certainty works best $\left(85 \% \mathrm{~F}_{1}\right)$ followed by attention $\left(82 \% \mathrm{~F}_{1}\right)$. The lowest performance is seen for control $\left(55 \% \mathrm{~F}_{1}\right)$ and circumstance $\left(61 \% \mathrm{~F}_{1}\right)$. These results are only partially in line with the inter-annotator agreement scores. We obtain a .75 micro average $F_{1}$ score.

Experiment 2 (Appraisal Interpretation, $\mathbf{A} \rightarrow \mathbf{E}$ ) aims at understanding how well emotions can be predicted from appraisals. We compare the baseline text-to-emotion setting $(\mathrm{T} \rightarrow \mathrm{E})$ to the pipeline setting that first predicts the appraisal and then, from those, the emotion. In the pipeline setting we train the second step $(\mathrm{A} \rightarrow \mathrm{E})$ on the gold appraisal annotations, not on the predictions. ${ }^{5}$ We compare this setting to the performance of the appraisal-to-emotion model $(\mathrm{A} \rightarrow \mathrm{E})$, when applied on gold appraisal annotations. This serves as an upper bound which can be reached with the best-performing appraisal prediction model.

We first turn to the results of the model which predicts the emotion based on annotated (gold) appraisal dimensions $(\mathrm{A} \rightarrow \mathrm{E}$ (gold)). Here, we observe a clear improvement in contrast to the emotion classification which has access to the text $(\mathrm{T} \rightarrow \mathrm{E})$. Anger increases from .52 to .62 , disgust decreases from .64 to .51 , fear increases from .70 to .78 , guilt from .44 to .63 , joy from .77 to .96 , sadness decreases from .68 to .66 and shame decreased from .45 to .43 . On micro average, the performance increases from .60 to $.66 \% \mathrm{~F}_{1}$. These results are an upper-bound for the performance that can be achieved with the pipeline model, under the assumption of having access to perfect appraisal predictions.

When moving to the real-world setting of first predicting the appraisal dimensions and then, based on those, predicting the emotion, the performance scores drop from 66 to $48 \% \mathrm{~F}_{1}$. This is an indicator

\footnotetext{
${ }^{5} \mathrm{We}$ also tested if training on the prediction leads to better results, but it constantly underperformed.
} 


\begin{tabular}{|c|c|c|c|c|c|c|c|c|c|}
\hline & \multirow[b]{2}{*}{ Emotion (G/P) } & \multicolumn{7}{|c|}{ Appraisal } & \multirow[b]{2}{*}{ Text } \\
\hline & & A & $\mathrm{Ce}$ & $\mathrm{E}$ & $\mathrm{P}$ & $\mathrm{R}$ & Co & $\mathrm{Ci}$ & \\
\hline \multirow{7}{*}{ 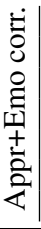 } & Anger & 1 & 1 & 0 & 0 & 0 & 0 & 0 & when my neighbour started to throw rubbish in my garden for no reason. \\
\hline & Disgust & 0 & 1 & 0 & 0 & 0 & 0 & 0 & to watch someone eat insects on television. \\
\hline & Fear & 1 & 0 & 1 & 0 & 0 & 0 & 1 & when our kitten escaped in the late evening and we thought he was lost. \\
\hline & Guilt & 0 & 1 & 0 & 0 & 1 & 1 & 0 & when I took something without paying. \\
\hline & Joy & 1 & 1 & 0 & 1 & 1 & 0 & 0 & when I found a rare item I had wanted for a long time. \\
\hline & Sadness & 1 & 1 & 1 & 0 & 0 & 0 & 1 & when my dog died. He was ill for a while. Still miss him. \\
\hline & Shame & 0 & 1 & 0 & 0 & 1 & 0 & 0 & when I remember an embarrassing social faux pas from my teenage years. \\
\hline \multirow{6}{*}{ 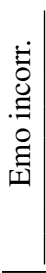 } & Anger/Fear & 1 & 0 & 1 & 0 & 0 & 0 & 0 & $\begin{array}{l}\text { when someone drove into my car causing damage and fear to myself - then } \\
\text { drove off before exchanging insurance details. }\end{array}$ \\
\hline & Disgust/Anger & 1 & 1 & 0 & 0 & 0 & 0 & 0 & when I saw a bird being mistreated when on holiday. \\
\hline & Fear/Sadness & 1 & 1 & 1 & 0 & 0 & 0 & 1 & a huge spider just plopped on down on the sofa besides me, staring me out. \\
\hline & Guilt/Disgust & 0 & 1 & 0 & 0 & 0 & 0 & 0 & $\begin{array}{l}\text { when I watched a documentary that showed footage of farms of pigs and } \\
\text { chickens and as a meat eater I felt awful guilt at how they are treated. }\end{array}$ \\
\hline & Sadness/Anger & 1 & 1 & 0 & 0 & 0 & 0 & 0 & when I saw a group of homeless people and it was cold outside. \\
\hline & Shame/Guilt & 0 & 1 & 0 & 0 & 1 & 1 & 0 & because I did something silly. \\
\hline \multirow{7}{*}{ 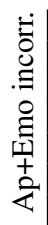 } & Anger/Shame & $\mathbf{0}$ & 1 & 0 & 0 & 1 & 0 & 0 & I feel ... because I can't stand when people lie. \\
\hline & Disgust/Anger & 1 & 1 & 0 & 0 & 0 & $\mathbf{0}$ & 0 & when I saw a medical operation on a TV show. \\
\hline & Fear/Guilt & 1 & 0 & $\mathbf{0}$ & 0 & 1 & 0 & $\mathbf{0}$ & when I was on a flight as I am ... of flying. \\
\hline & Guilt/Shame & 0 & 1 & 1 & 0 & 1 & 1 & 0 & when I lost my sister's necklace that I had borrowed. \\
\hline & Joy/Anger & 1 & 1 & 0 & $\mathbf{0}$ & 0 & 0 & $\mathbf{0}$ & when I saw bees coming back to my garden after few years of absence. \\
\hline & Sadness/Guilt & 1 & 1 & 0 & 0 & 1 & 0 & $\mathbf{0}$ & when I watched some of the sad cases of children in need. \\
\hline & Shame/Guilt & 0 & 1 & 0 & 0 & 1 & 1 & 0 & when I forgot a hairdressers appointment. \\
\hline
\end{tabular}

Table 6: Examples for the prediction of the pipeline setting $(\mathrm{T} \rightarrow \mathrm{A}, \mathrm{A} \rightarrow \mathrm{E})$. A: Attention, Ce: Certainty, E: Effort, P: Pleasantness, R: Responsibility, Co: Control, Ci: Circumstance. First emotion mention is gold, second is prediction. Appraisal shown is prediction with errors shown in bold.

that the performance of our current appraisal prediction, though comparably reasonable with $75 \% \mathrm{~F}_{1}$, is not yet sufficient to support emotion predictions, at least partially. The clear improvement in emotion prediction based on perfect appraisal annotations and the performance drop in the real-world setting for emotion prediction suggest that annotating more data with appraisal dimensions is necessary to further develop our approach.

Finally, Experiment 3 (Multi-Task Learning, $\mathbf{T} \rightarrow \mathbf{A} / \mathbf{E}$ and Oracle Ensembles) evaluates if the model which learns appraisals and emotions jointly performs better than the pipeline model (both being models applicable in a real-world scenario). We show these results also in Table 5 and see that the multi-task learning model cannot improve over the text-only setting.

A remaining question is on the complementarity of the pipeline and the multitask model to the $\mathrm{T} \rightarrow \mathrm{E}$ model. To look into this, we define two oracle ensembles $(\mathrm{T} \rightarrow \mathrm{A}, \mathrm{A} \rightarrow \mathrm{E}$ and $\mathrm{T} \rightarrow \mathrm{E}$ as well as $\mathrm{T} \rightarrow \mathrm{A} / \mathrm{E}$ and $\mathrm{T} \rightarrow \mathrm{E}$ ), in which an oracle predicts which of the two approaches will obtain the correct result. In this experiment, we therefore accept a prediction as true positive if one of the two parts of the ensemble is correct. These results are shown in Table 5 in the columns "Oracle Ensemble". We see a clear improvement over the isolated text-based prediction for both oracle ensembles, while the pipeline model shows a higher contribution in addition to the $\mathrm{T} \rightarrow \mathrm{E}$ model $\left(70 \% \mathrm{~F}_{1}\right.$ in contrast to $\left.66 \% \mathrm{~F}_{1}\right)$.

\subsection{Discussion and Analysis}

We have seen in the experiments and results that the approach of predicting emotions based on appraisal shows a clear potential for a better performance. Though we have not been able to reach a substantial improvement in a real-world setting, in which appraisal dimensions are first predicted as a basis for the emotion prediction or in the multi-task setting, we observe that text- and appraisal-based models behave differently. Table 6 shows examples for the prediction in the real-world setting $(T \rightarrow A, A \rightarrow E)$. In the top block, one example is shown for each emotion in which both appraisal and emotion are correctly predicted. This does not only include cases in which clear emotion indicators exist. The second block reports instances in which the appraisal is correct, but the emotion prediction is not. Here, the first sentence ("when someone drove into my car...") is an example in which a flip of certainty would have changed the emotion. Similarly, the change of attention in "when I saw a group..." would have lead 


\begin{tabular}{|c|c|c|c|}
\hline Gold Emotion & $\mathrm{A} \rightarrow \mathrm{E}$ & $\mathrm{T} \rightarrow \mathrm{E}$ & Text \\
\hline Anger & Anger & Fear & because I was overlooked at work. \\
\hline Anger & Anger & Disgust & when I saw someone mistreating an animal. \\
\hline Anger & Anger & Fear & when someone overtook my car on a blind bend and nearly caused an accident. \\
\hline Disgust & Disgust & Shame & because I ate a sausage that was horrible. \\
\hline Disgust & Disgust & Fear & when I was on a ferry in a storm and lots of people were vomiting. \\
\hline Disgust & Disgust & Shame & because the milk I put in my coffee had lumps in it. \\
\hline Fear & Fear & Shame & because I had to have a general anaesthetic for an operation. \\
\hline Fear & Fear & Sadness & when my 2 year old broke her leg, and we felt helpless to assist her. \\
\hline Fear & Fear & Anger & $\begin{array}{l}\text { because we were driving fast in the rain in order to get somewhere before it shut, } \\
\text { and the driver was going over the speed limit. }\end{array}$ \\
\hline Guilt & uilt & ame & \\
\hline Guilt & Guilt & & for denying to offer my kids what they demanded \\
\hline Guilt & Guilt & Anger & when I had not done a job for a friend that I had promised to do. \\
\hline Joy & Joy & Sadness & when witnessing the joy on my children's face on Christmas morning. \\
\hline Joy & Joy & Shame & when I managed to complete a cryptic crossword. \\
\hline Joy & Joy & Disgust & when I found a twenty pound note on the ground outside. \\
\hline Sadness & Sadness & Fear & when it was raining this morning as I been planning to go o \\
\hline Sadness & Sadness & Joy & $\begin{array}{l}\text { I feel ... when I see the Christmas decorations come down, and know they won't } \\
\text { be up again for another year. }\end{array}$ \\
\hline Sadness & Sadness & Shame & $\begin{array}{l}\text { when my friend's eye was watering after an injection into it and I could do } \\
\text { nothing to help. }\end{array}$ \\
\hline Shat & hame & & when I failed my ninth year at high school. \\
\hline Shame & Shame & Guilt & when I had too much to drink in a pub, fell over and had to go to hospital. \\
\hline Shame & Shame & Anger & when my mom caught me lying. \\
\hline
\end{tabular}

Table 7: Examples in which the appraisal model (on gold appraisal annotation) predicts the correct emotion and the baseline system does not.

to the correct emotion prediction. These are therefore untypical cases of appraisal assignment. The last block shows examples where the wrong appraisal prediction leads to wrong emotion assignment.

It is further interesting to look into those cases which are wrongly predicted from text, but correctly predicted based on the gold appraisal annotations. We show examples for such cases in Table 7. Several of these cases are examples in which a word seems to indicate a particular emotion, which is actually not relevant to infer the emotion in the first place (e.g., "animal", "vomiting", "kids", "high school"). Often, shame is wrongly predicted when the event is about the self. This is particularly problematic if the actual word pointing to an emotion appears to be non-typical (e.g., "crossword", "anaesthetic").

\section{Conclusions and Future Work}

We investigated the hypothesis that informing an emotion classification model about the cognitive appraisal regarding a situation is beneficial for the model performance. We were able to show that emotion classification performs better than text-based classification, under the assumption that perfect appraisal predictions are possible and shows complementary correct predictions. Yet, neither in a multi-task learning nor a pipeline, in which the appraisal was predicted as a basis, could we show an improvement in emotion classification. This provides evidence that, though our appraisal predictor is of reasonable performance, the model suffers from error propagation. This is still an encouraging result, suggesting that future work should further investigate the combination of appraisal information with emotion prediction, particularly in the light of our oracle ensemble that indicated a clear improvement.

This first study on the topic raises a couple of research questions: Would there be other neural architectures which are better suited for including the appraisal information? Will more annotated data improve the prediction quality sufficiently? Finally, it should be analyzed if giving the annotators access to the emotion label when making the appraisal annotation could have changed the results.

\section{Acknowledgements}

This work was supported by Leibniz WissenschaftsCampus Tübingen "Cognitive Interfaces" and Deutsche Forschungsgemeinschaft (project SEAT, KL 2869/1). We thank Laura Ana Maria Oberländer for inspiration and fruitful discussions and Valentino Sabbatino for his annotation work. Further, we thank the three anonymous reviewers for their constructive and helpful criticism. 


\section{References}

Muhammad Abdul-Mageed and Lyle Ungar. 2017. EmoNet: Fine-grained emotion detection with gated recurrent neural networks. In Proceedings of the 55th Annual Meeting of the Association for Computational Linguistics (Volume 1: Long Papers), pages 718-728, Vancouver, Canada, July. Association for Computational Linguistics.

Cecilia Ovesdotter Alm, Dan Roth, and Richard Sproat. 2005. Emotions from text: Machine learning for textbased emotion prediction. In Proceedings of Human Language Technology Conference and Conference on Empirical Methods in Natural Language Processing, pages 579-586, Vancouver, British Columbia, Canada. Association for Computational Linguistics.

Saima Aman and Stan Szpakowicz. 2007. Identifying expressions of emotion in text. In International Conference on Text, Speech and Dialogue, pages 196-205. Springer.

James R Averill. 1980. A constructivist view of emotion. In Theories of emotion, pages 305-339. Elsevier.

Alexandra Balahur, Jesús M. Hermida, Andrés Montoyo, and Rafael Muñoz. 2011. Emotinet: A knowledge base for emotion detection in text built on the appraisal theories. In Rafael Muñoz, Andrés Montoyo, and Elisabeth Métais, editors, Natural Language Processing and Information Systems, pages 27-39, Berlin, Heidelberg. Springer Berlin Heidelberg.

Alexandra Balahur, Jesus M. Hermida, and Andrew Montoyo. 2012. Building and exploiting emotinet, a knowledge base for emotion detection based on the appraisal theory model. IEEE Transactions on Affective Computing, 3(1):88-101.

Lisa Feldman Barrett and James A Russell. 2015. The psychological construction of emotion. Guilford Publications, New York.

Laura-Ana-Maria Bostan and Roman Klinger. 2018. An analysis of annotated corpora for emotion classification in text. In Proceedings of the 27th International Conference on Computational Linguistics, pages 2104-2119, Santa Fe, New Mexico, USA. Association for Computational Linguistics.

Laura Ana Maria Bostan, Evgeny Kim, and Roman Klinger. 2020. GoodNewsEveryone: A corpus of news headlines annotated with emotions, semantic roles, and reader perception. In Nicoletta Calzolari, Khalid Choukri, Thierry Declerck, Hrafn Loftsson, Bente Maegaard, Joseph Mariani, Asuncion Moreno, Jan Odijk, and Stelios Piperidis, editors, Proceedings of the 12th International Conference on Language Resources and Evaluation (LREC'20), Marseille, France. European Language Resources Association (ELRA).

Joost Broekens, Doug DeGroot, and Walter A. Kosters. 2008. Formal models of appraisal: Theory, specification, and computational model. Cognitive Systems Research, 9(3):173 - 197.

Sven Buechel and Udo Hahn. 2017. EmoBank: Studying the impact of annotation perspective and representation format on dimensional emotion analysis. In Proceedings of the 15th Conference of the European Chapter of the Association for Computational Linguistics: Volume 2, Short Papers, pages 578-585, Valencia, Spain. Association for Computational Linguistics.

Sven Buechel, Johannes Hellrich, and Udo Hahn. 2016. Feelings from the Past_-Adapting affective lexicons for historical emotion analysis. In Proceedings of the Workshop on Language Technology Resources and Tools for Digital Humanities (LT4DH), pages 54-61, Osaka, Japan, December. The COLING 2016 Organizing Committee.

Andres Campero, Bjarke Felbo, Joshua B. Tenenbaum, and Rebecca Saxe. 2017. A first step in combining cognitive event features and natural language representations to predict emotions. In Conference on Cognitive Computational Neuroscience.

Deniz Cevher, Sebastian Zepf, and Roman Klinger. 2019. Towards multimodal emotion recognition in german speech events in cars using transfer learning. In Proceedings of the 15th Conference on Natural Language Processing (KONVENS 2019): Long Papers, pages 79-90, Erlangen, Germany. German Society for Computational Linguistics \& Language Technology.

Gerald. L. Clore and Andrew Ortony. 2013. Psychological Construction in the OCC Model of Emotion. Emotion Review, 5(4):335-343.

Paul Ekman. 1992. An argument for basic emotions. Cognition \& emotion, 6(3-4):169-200.

Paul Ekman. 1999. Basic emotions. In Tim Dalgleish and Mick J. Power, editors, Handbook of Cognition and Emotion. John Wiley \& Sons, Sussex, UK. 
Bjarke Felbo, Alan Mislove, Anders Søgaard, Iyad Rahwan, and Sune Lehmann. 2017. Using millions of emoji occurrences to learn any-domain representations for detecting sentiment, emotion and sarcasm. In Proceedings of the 2017 Conference on Empirical Methods in Natural Language Processing, pages 1615-1625, Copenhagen, Denmark. Association for Computational Linguistics.

Lisa Feldman Barrett. 2006. Solving the emotion paradox: categorization and the experience of emotion. Personality and Social Psychology Review, 10(1):20-46.

Maria Gendron, Debi Roberson, Jacoba Marietta van der Vyver, and Lisa Feldman Barrett. 2014. Perceptions of emotion from facial expressions are not culturally universal: evidence from a remote culture. Emotion, 14(2):251-262.

Carroll E. Izard. 1971. The face of emotion. Appleton-Century-Crofts, New York.

Evgeny Kim, Sebastian Padó, and Roman Klinger. 2017. Investigating the relationship between literary genres and emotional plot development. In Proceedings of the Joint SIGHUM Workshop on Computational Linguistics for Cultural Heritage, Social Sciences, Humanities and Literature, pages 17-26, Vancouver, Canada. Association for Computational Linguistics.

Yoon Kim. 2014. Convolutional neural networks for sentence classification. In Proceedings of the 2014 Conference on Empirical Methods in Natural Language Processing (EMNLP), pages 1746-1751, Doha, Qatar. Association for Computational Linguistics.

Roman Klinger, Orphée De Clercq, Saif Mohammad, and Alexandra Balahur. 2018. IEST: WASSA-2018 implicit emotions shared task. In Proceedings of the 9th Workshop on Computational Approaches to Subjectivity, Sentiment and Social Media Analysis, pages 31-42, Brussels, Belgium. Association for Computational Linguistics.

Yanran Li, Hui Su, Xiaoyu Shen, Wenjie Li, Ziqiang Cao, and Shuzi Niu. 2017. DailyDialog: A manually labelled multi-turn dialogue dataset. In Proceedings of the Eighth International Joint Conference on Natural Language Processing (Volume 1: Long Papers), pages 986-995, Taipei, Taiwan. Asian Federation of Natural Language Processing.

Stacy C. Marsella and Jonathan Gratch. 2009. EMA: A process model of appraisal dynamics. Cognitive Systems Research, 10(1):70 - 90. Modeling the Cognitive Antecedents and Consequences of Emotion.

Stacy C. Marsella, Jonathan Gratch, and Paolo Petta. 2010. Computational models of emotion. In Klaus R. Scherer, Tanja Bänziger, and Etienne Roesch, editors, A Blueprint for Affective Computing. Oxford University Press.

Saif Mohammad and Felipe Bravo-Marquez. 2017. WASSA-2017 shared task on emotion intensity. In Proceedings of the 8th Workshop on Computational Approaches to Subjectivity, Sentiment and Social Media Analysis, pages 34-49, Copenhagen, Denmark. Association for Computational Linguistics.

Saif M. Mohammad and Peter D. Turney. 2012. Crowdsourcing a word-emotion association lexicon. Computational Intelligence, 29(3).

Saif M. Mohammad, Parinaz Sobhani, and Svetlana Kiritchenko. 2017. Stance and sentiment in tweets. ACM Trans. Internet Technol., 17(3).

Saif Mohammad, Felipe Bravo-Marquez, Mohammad Salameh, and Svetlana Kiritchenko. 2018. SemEval-2018 task 1: Affect in tweets. In Proceedings of The 12th International Workshop on Semantic Evaluation, pages 1-17, New Orleans, Louisiana. Association for Computational Linguistics.

Saif Mohammad. 2012. \#emotional tweets. In *SEM 2012: The First Joint Conference on Lexical and Computational Semantics - Volume 1: Proceedings of the main conference and the shared task, and Volume 2: Proceedings of the Sixth International Workshop on Semantic Evaluation (SemEval 2012), pages 246-255, Montréal, Canada. Association for Computational Linguistics.

Saif M. Mohammad. 2018. Obtaining reliable human ratings of valence, arousal, and dominance for 20,000 english words. In Proceedings of The Annual Conference of the Association for Computational Linguistics $(A C L)$, Melbourne, Australia.

Vinod Nair and Geoffrey E. Hinton. 2010. Rectified linear units improve restricted Boltzmann machines. In Johannes Fürnkranz and Thorsten Joachims, editors, Proceedings of the 27th International Conference on Machine Learning (ICML-10), pages 807-814, Haifa, Israel. Omnipress.

Keith Oatley and Philip N. Johnson-Laird. 1987. Towards a cognitive theory of emotions. Cognition and emotion, 1(1):29-50. 
Keith Oatley. 1993. Social construction in emotions. In M. Lewis and J. M. Haviland, editors, Handbook of emotions, pages 341-352. Guilford Press, New York.

James W Pennebaker, Martha E Francis, and Roger J Booth. 2001. Linguistic inquiry and word count: Liwc 2001. Mahway: Lawrence Erlbaum Associates, 71:2001.

Jeffrey Pennington, Richard Socher, and Christopher Manning. 2014. Glove: Global vectors for word representation. In Proceedings of the 2014 Conference on Empirical Methods in Natural Language Processing (EMNLP), pages 1532-1543, Doha, Qatar. Association for Computational Linguistics.

Robert Plutchik. 2001. The nature of emotions human emotions have deep evolutionary roots, a fact that may explain their complexity and provide tools for clinical practice. American Scientist, 89(4):344-350.

Jonathan Posner, James A. Russell, and Bradley S. Peterson. 2005. The circumplex model of affect: an integrative approach to affective neuroscience, cognitive development, and psychopathology. Development and Psychopathology, 17(3):715-734.

Daniel Preotiuc-Pietro, H. Andrew Schwartz, Gregory Park, Johannes Eichstaedt, Margaret Kern, Lyle Ungar, and Elisabeth Shulman. 2016. Modelling valence and arousal in Facebook posts. In Proceedings of the 7th Workshop on Computational Approaches to Subjectivity, Sentiment and Social Media Analysis, pages 9-15, San Diego, California. Association for Computational Linguistics.

Klaus R. Scherer and Harald G. Wallbott. 1997. The ISEAR questionnaire and codebook. Geneva Emotion Research Group. https://www.unige.ch/cisa/research/ materials-and-online-research/research-material/.

Klaus R. Scherer, Angela Schorr, and Tom Johnstone. 2001. Appraisal processes in emotion: Theory, methods, research. Oxford University Press.

Klaus R. Scherer, Tanja Bänziger, and Etienne Roesch. 2010. A Blueprint for Affective Computing. Oxford University Press.

Klaus R. Scherer, Heiner Ellgring, Anja Dieckmann, Matthias Unfried, and Marcello Mortillaro. 2019. Dynamic facial expression of emotion and observer inference. Frontiers in Psychology, 10:508.

Klaus R. Scherer. 1982. Emotion as a process: Function, origin and regulation. Social Science Information, 48:555-70.

Klaus R. Scherer. 2005. What are emotions? And how can they be measured? Social Science Information, 44(4):695-729.

Hendrik Schuff, Jeremy Barnes, Julian Mohme, Sebastian Padó, and Roman Klinger. 2017. Annotation, modelling and analysis of fine-grained emotions on a stance and sentiment detection corpus. In Proceedings of the 8th Workshop on Computational Approaches to Subjectivity, Sentiment and Social Media Analysis, pages 13-23, Copenhagen, Denmark. Association for Computational Linguistics.

Mostafa Al Masum Shaikh, Helmut Prendinger, and Mitsuru Ishizuka. 2009. A linguistic interpretation of the occ emotion model for affect sensing from text. Affective Information Processing, pages 45-73.

Craig. A. Smith and Phoebe. C. Ellsworth. 1985. Patterns of cognitive appraisal in emotion. Journal of Personality and Social Psychology, 48(4):813-838.

Carlo Strapparava and Rada Mihalcea. 2007. SemEval-2007 task 14: Affective text. In Proceedings of the Fourth International Workshop on Semantic Evaluations (SemEval-2007), pages 70-74, Prague, Czech Republic, June. Association for Computational Linguistics.

Carlo Strapparava and Alessandro Valitutti. 2004. WordNet affect: an affective extension of WordNet. In Proceedings of the Fourth International Conference on Language Resources and Evaluation (LREC'04), Lisbon, Portugal. European Language Resources Association (ELRA).

John Tooby and Leda Cosmides. 2008. The evolutionary psychology of the emotions and their relationship to internal regulatory variables. In J.M. Haviland-Jones M. Lewis and L. F. Barrett, editors, Handbook of emotions, pages 114-137. Guilford Press, New York.

Jessica L. Tracy and Richard W. Robins. 2006. Appraisal antecedents of shame and guilt: Support for a theoretical model. Personality and social psychology bulletin, 32(10):1339-1351.

Enrica Troiano, Sebastian Padó, and Roman Klinger. 2019. Crowdsourcing and validating event-focused emotion corpora for German and English. In Proceedings of the 57th Annual Meeting of the Association for Computational Linguistics, pages 4005-4011, Florence, Italy. Association for Computational Linguistics. 


\section{A Appraisal Prediction Performance of the Multitask Model}

We focused in the discussion of the results in Section 4.2 on the goal to improve the performance of the emotion prediction with appraisal information. Though we evaluated the appraisal prediction model as an intermediate step in the pipeline to emotion prediction, we did only evaluate the emotion performance in the multitask learning setting due to our focus on emotion categorization.

However, it would be meaningful to also experiment with a $\mathrm{T} \rightarrow \mathrm{E}, \mathrm{E} \rightarrow \mathrm{A}$ model when the goal would be to predict the appraisal dimensions with or without knowledge of an emotion category. We leave such evaluation for future work. However, our multitask model $\mathrm{T} \rightarrow \mathrm{A} / \mathrm{E}$ also produces appraisal predictions in the context of emotion predictions. We therefore provide the appraisal evaluation of the $\mathrm{T} \rightarrow \mathrm{A} / \mathrm{E}$ model in Table 8 . We see that the appraisal prediction does not show any improvement over the $\mathrm{T} \rightarrow \mathrm{A}$ model with the respective results in Table 4.

\begin{tabular}{lllll}
\hline & & \multicolumn{3}{c}{$\mathrm{T} \rightarrow \mathrm{A} / \mathrm{E}$} \\
\cline { 4 - 6 } Appraisal & & $\mathrm{P}$ & $\mathrm{R}$ & $\mathrm{F}_{1}$ \\
\cline { 1 - 3 } Attention & & 82 & 78 & 80 \\
Certainty & & 85 & 76 & 80 \\
Effort & & 65 & 62 & 64 \\
Pleasantness & & 76 & 59 & 67 \\
Responsibility & & 71 & 69 & 70 \\
Control & & 60 & 42 & 49 \\
Circumstance & & 67 & 48 & 56 \\
\cline { 1 - 3 } Macro avg. & & 72 & 62 & 67 \\
Micro avg. & & 76 & 68 & 71 \\
\hline
\end{tabular}

Table 8: Multi-task learning model performance on predicting appraisal dimensions.

\section{B Examples from the Multitask Model}

We show examples from the $\mathrm{T} \rightarrow \mathrm{A} / \mathrm{E}$ model in Table 9 , similarly to the results from $\mathrm{T} \rightarrow \mathrm{A}, \mathrm{A} \rightarrow \mathrm{E}$ examples in Table 6. Note that, given the bidirectional interdependencies between emotion and appraisal taking place in the multitask learning, in contrast to the unidirectional information flow from appraisal to emotion in the pipeline, these results are more challenging to interpret.

\begin{tabular}{|c|c|c|c|c|c|c|c|c|c|}
\hline \multirow{9}{*}{ 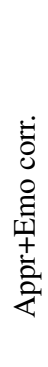 } & \multirow[b]{2}{*}{ Emotion (G/P) } & \multicolumn{7}{|c|}{ Appraisal } & \multirow[b]{2}{*}{ Text } \\
\hline & & \multicolumn{7}{|c|}{$\mathrm{R}$ Co Ci } & \\
\hline & Anger & 1 & 1 & 0 & 0 & 0 & 0 & 0 & when I heard someone gossip about a friend of mine. \\
\hline & Disgust & 0 & 1 & 0 & 0 & 0 & & 1 & because the drain smelled. \\
\hline & Fear & 1 & 0 & 1 & 0 & 0 & 0 & 0 & when I thought my son could be in trouble when mountain climbing. \\
\hline & Guilt & 0 & 1 & 0 & 0 & 1 & 1 & 0 & because I drank way too much, knowing that I had things to do the next day. \\
\hline & Joy & 1 & 1 & 0 & 1 & 0 & 0 & 0 & because my son gave me a big hug when I got home from work. \\
\hline & Sadness & 1 & 1 & 0 & 0 & 0 & 0 & 0 & when I read about orangutans and palm oil \\
\hline & Shame & 0 & 1 & 0 & 0 & 1 & & 0 & $\begin{array}{l}\text { because I walked past a beggar and didn't bother to stop and give her a } \\
\text { pound. }\end{array}$ \\
\hline \multirow{7}{*}{ 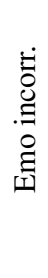 } & Anger/Shame & 1 & 0 & 0 & 0 & 0 & 0 & 0 & because the parents dropping their kids off to school were driving and parking \\
\hline & Anger/Disgust & 1 & 0 & 1 & 0 & 0 & 0 & 0 & someone stabbed a man on a train. \\
\hline & Disgust/Sadness & 1 & 1 & 0 & 0 & 0 & 0 & 0 & when I read that hunters had killed one of the world famous lions. \\
\hline & Disgust/Anger & 1 & 1 & 0 & 0 & 0 & 0 & 0 & when I watched videos of how some people treat animals. \\
\hline & Guilt/Shame & 0 & 1 & 0 & 0 & 1 & 1 & 0 & because I cheated on a previous girlfriend. \\
\hline & Sadness/Anger & 1 & 1 & 0 & 0 & 0 & 0 & 0 & when listening to the national news. \\
\hline & Shame/Guilt & 0 & 1 & 0 & 0 & 1 & 1 & 0 & when my daughter was rude to my wife. \\
\hline \multirow{7}{*}{ 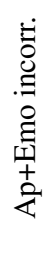 } & Anger/Disgust & 1 & 1 & $\mathbf{0}$ & 0 & 0 & 0 & 0 & $\mathrm{n}$ \\
\hline & Disgust/Fear & 1 & 1 & $\mathbf{0}$ & 0 & 1 & 0 & $\mathbf{0}$ & stole $\mathrm{m}$ \\
\hline & Fear/Shame & $\mathbf{0}$ & 1 & $\mathbf{0}$ & 0 & 1 & 0 & 0 & because a fight broke out in a bar where I was $d_{1}$ \\
\hline & Guilt/Disgust & $\mathbf{0}$ & 1 & $\mathbf{0}$ & 0 & $\mathbf{0}$ & 0 & 0 & $\begin{array}{l}\text { because I accidentally killed my pet fish by decorating his tank with untreated } \\
\text { sand. }\end{array}$ \\
\hline & Joy/Sadness & 1 & 1 & 0 & $\mathbf{0}$ & $\mathbf{0}$ & $\mathbf{0}$ & 0 & when I rescued a dog at the local shelter and he became my best friend. \\
\hline & Sadness/Joy & 1 & 1 & $\mathbf{0}$ & 0 & 0 & 0 & 1 & when I was on holiday and it was the last day and had to come home. \\
\hline & Shame/Guilt & 0 & 1 & 0 & 0 & 1 & $\mathbf{0}$ & 1 & en I ate my whole Easter egg in one go. \\
\hline
\end{tabular}

Table 9: Examples for the prediction of the multi-task setting $(\mathrm{T} \rightarrow \mathrm{A} / \mathrm{E})$. A: Attention, Ce: Certainty, E: Effort, P: Pleasantness, R: Responsibility, Co: Control, Ci: Circumstance. First emotion mention is gold, second is prediction. Appraisal shown is prediction with errors shown in bold. 